\title{
Split striplines with adjustable parameters and devices based on them
}

\author{
Nickolay Malyutin ${ }^{1, *}$, Alexandra Malyutina ${ }^{2}$, Georgiy Malyutin ${ }^{1}$, and Alexander Zabolotsky ${ }^{1}$ \\ ${ }^{1}$ Tomsk State University of Control Systems and Radioelectronics, Ave. Lenin, 40, Tomsk, 634050, \\ Russia \\ ${ }^{2}$ National Research Tomsk State University Ave. Lenin, 36, Tomsk, 634050, Russia
}

\begin{abstract}
The split strip lines (SSL) with adjustable parameters and examples of devices based on them are examined. Changing the parameters of the split strip lines are carried out with the help of so-called controlling strips, coupled with parts of the split strip line and having a strong electromagnetic coupling with them. Between controlling strips and the screen the lumped regulating elements are included. In wide frequency range such strip structures can be considered as transmission lines with changeable characteristic impedance and changeable propagation coefficient. Frequency analysis of of scattering matrix $\mathrm{S}$ of controlled split strip lines with different circuits of strip inclusion is carried out. Examples of the use of split strip lines with adjustable parameters in the design of different devices were discussed.
\end{abstract}

\section{Introduction}

There are two main methods to change the parameters of striplines and coupled strip transmission lines. The first method is based on control of dielectric parameters, magnetic or magneto-dielectric medium [1-5]. The second method is based on the introduction of lumped heterogeneities, creating the effect of changing parameters in a limited frequencies range, as this heterogeneity creates a frequency-selective circuit [6]. Segnetoelectric capacitors and varicaps are used as a lumped controlled heterogeneities.

Transmission lines do not change their properties when the control elements are included in them, and the effect of changing the parameters of the system is observed due to interference of incident and reflected waves from inhomogeneities.

The change of parameters in the controlled sections of the coupled strip lines was shown in the work [6]. Expressions were obtained for calculation of frequency characteristics of the controlled sections at variation of impedance of the regulating elements. At the same time, the section of coupled lines is considered as a strip line with a changeable characteristic impedance and a changeable propagation factor, if the dielectric filling of the structure is heterogeneous in the cross section.

In the present work we consider so-called split strip lines (SSL), which are formed by dividing a current-carrying conductor with a longitudinal gap into two equal parts. The task was to study the frequency properties of SSL and the possibilities of adjusting their parameters by introducing an additional strip conductor and lumped elements.

\footnotetext{
*Corresponding author: ndm@main.tusur.ru
} 


\section{Frequency characteristics of base sections}

Analysis and experimental studies were carried out on several schemes for switching on SSL conductors, shown in Fig. 1 - Fig. 6. Equivalent schemes of the shown base sections include strips 1 and 2, loads at the input and output of $Z_{\mathrm{L} 1}, Z_{\mathrm{L} 2}$ and voltage source $E$. Additional strips 3 are introduced into schemes 2, 3, 5 and 6 , having a strong electromagnetic coupling with strips 1 and 2 .

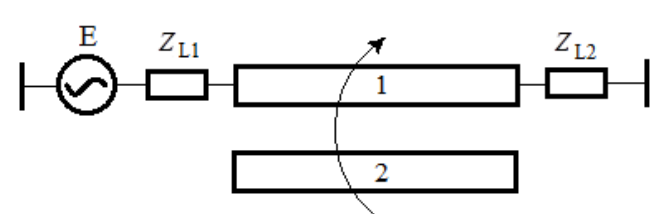

Fig. 1. Split strip line segment, in which the second strip is not loaded.

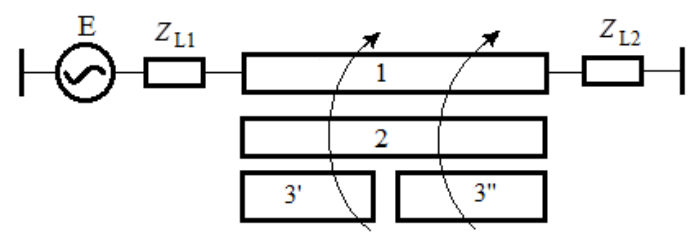

Fig. 3. Split strip line segment with inserted strip 3, having a strong electromagnetic coupling with strips 1 and 2 and divided by a transverse gap into 2 parts 3 "and 3".

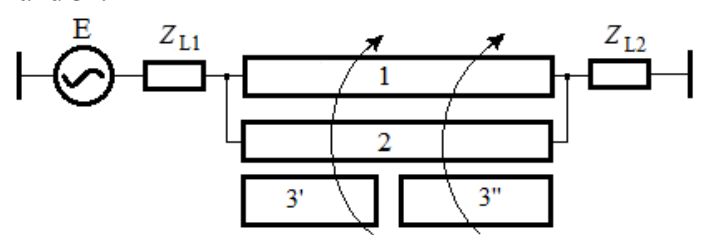

Fig. 5. Split strip line segment in which strips 1 and 2 are connected in parallel and a third strip is inserted and coupled with the 1'st and 2'nd strips and segmented by a transverse gap into 2 parts.

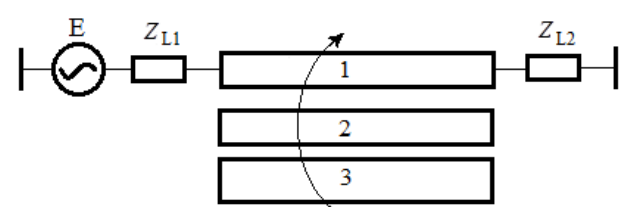

Fig. 2. Section of split strip line with inserted strip 3 having strong electromagnetic coupling with strips 1 and 2 .

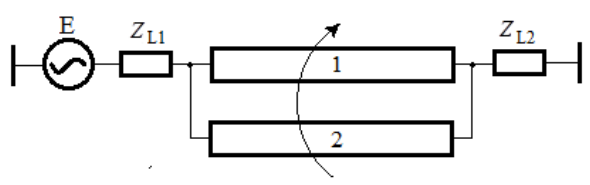

Fig. 4. Split strip line segment, strips 1 and 2 are connected in parallel.

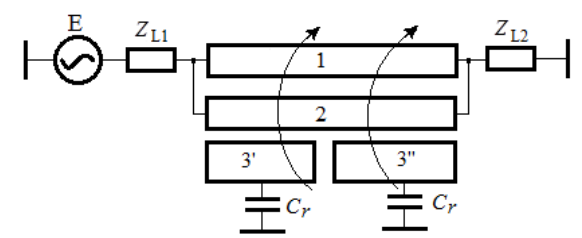

Fig. 6. Split strip line segment with adjustment of parameters by means of lumped elements with capacity $\mathrm{Cr}$.

Strips 1 and 2 in all diagrams represent two coupled lines in which quasi-T waves of inphase and anti-phase types are spread [7] despite the fact that their excitation at the point of powering of the scheme is close to in-phase (even). In general, these waves have different phase velocities, so interference of incident and reflected waves is observed in the SSL segment.

The SSL layout was made with the SSL cross-sectional dimensions shown in Fig. 7. The dimensions are $W=1,1 \mathrm{~mm}, D=1 \mathrm{~mm}, h=1,5 \mathrm{~mm}, A=10 \mathrm{~mm}, B=4 \mathrm{~mm}, \varepsilon_{1}=4,5$, $\varepsilon_{2}=1,0$. Calculation of matrixes of per unit length capacities $C$ and inductivities $L$ was carried out by means of grid method [8] and gave such values of elements $C$ and $L$ : $C_{11}=C_{22}=90,497 \mathrm{pF} / \mathrm{m}, C_{12}=C_{21}=-4,545 \mathrm{pF} / \mathrm{m}, L_{11}=L_{22}=0.375 \mu \mathrm{H} / \mathrm{m}$.

Fig. 8 shows the theoretically and experimentally obtained relationships of the insertion and return losses of the circuit of Fig. 1 to the ratio of the segment length to the wavelength of the acting signal in the free space. Dependences of $S_{11}$ and $S_{21}$ from $1 / \lambda$ show that in the limited frequency range SSL segment with the scheme of Fig. 1 is matched at the level of return losses $-10 \mathrm{~dB}$ and less than $1 \mathrm{~dB}$ inserted losses. An experiment was conducted to 
improve the parameters of the split strip line segment shown in Fig. 1 by adding a strip 3 (Fig. 2). The cross-section of the strip structure is shown in Fig. 9.

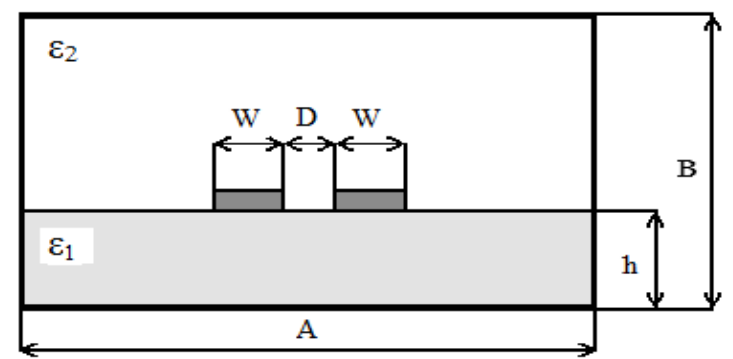

Fig. 7. Design cross-sectional SSL (strips 1 and 2).

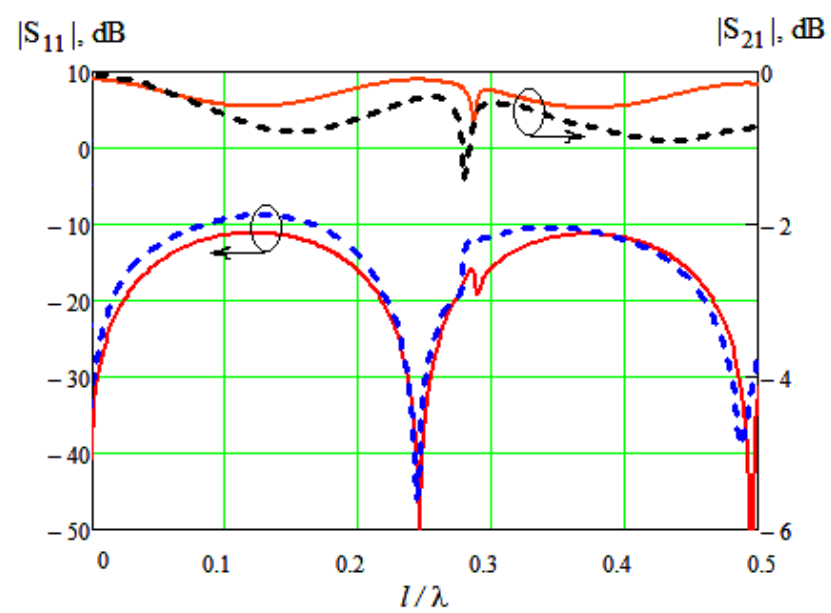

- Theorey $\left|\mathrm{S}_{11}\right|, \mathrm{dB} \quad$--- Experiment $\left|\mathrm{S}_{11}\right|_{, \mathrm{dB}}$

- Theorey $\left|S_{21}\right|, d B$ - -- Experiment $\left|S_{21}\right|, d B$

Fig. 8. Theoretical and experimental dependences of insertion and return loss of the SSL segment of the scheme (Fig. 1) at the ratio of the length to the wavelength of affecting signal in free space.

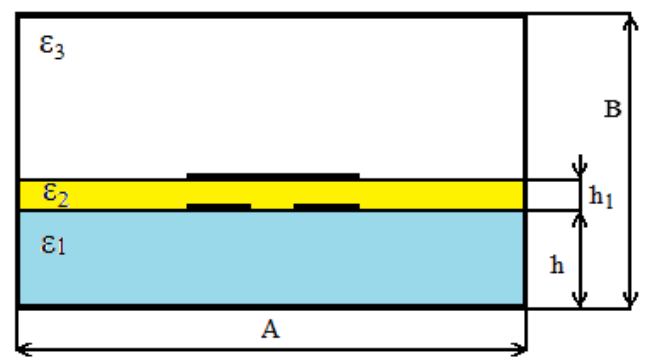

Fig. 9. The cross section of SSL with additional strip.

The impact on the insertion losses of the inserted strip 3 (diagram of Fig. 2) and its division into two parts by a transverse gap (diagram of Fig. 3) has been measured. The measurement results are still shown in Fig. 10.

Comparison of dependencies $S_{21}(1 / \lambda)$ shows a decrease in insertion loss following the result of the introduction of the strips 3 and its segmentation.

Calculation and experimental measurements of the diagram of Fig. 4 showed that the frequency characteristic of the SSL segment when the strips 1 and 2 are connected in 
parallel has pronounced resonances at the ratio of length of the segment $l$ to wavelength $\lambda$ of the acting signal in free space at $l / \lambda=0.13$ and $l / \lambda=0.40$ in the range of change of $l / \lambda=0 \ldots 0.5$ (Fig. 11). This result was unexpected because the strips 1 and 2 in the circuit are in-phase at the point where the signal source is turned on and the coupling between them is rather small.

Fig. 11 illustrates the change of the frequency dependence of insertion and return loss in the transition from the strip structure of Fig. 1 and Fig. 7 (experiment 1) to the circuit conductors of Fig. 5 and structure of Fig. 9 (experiment 2). We are observing the disappearance of resonances and a significant decrease in return loss.

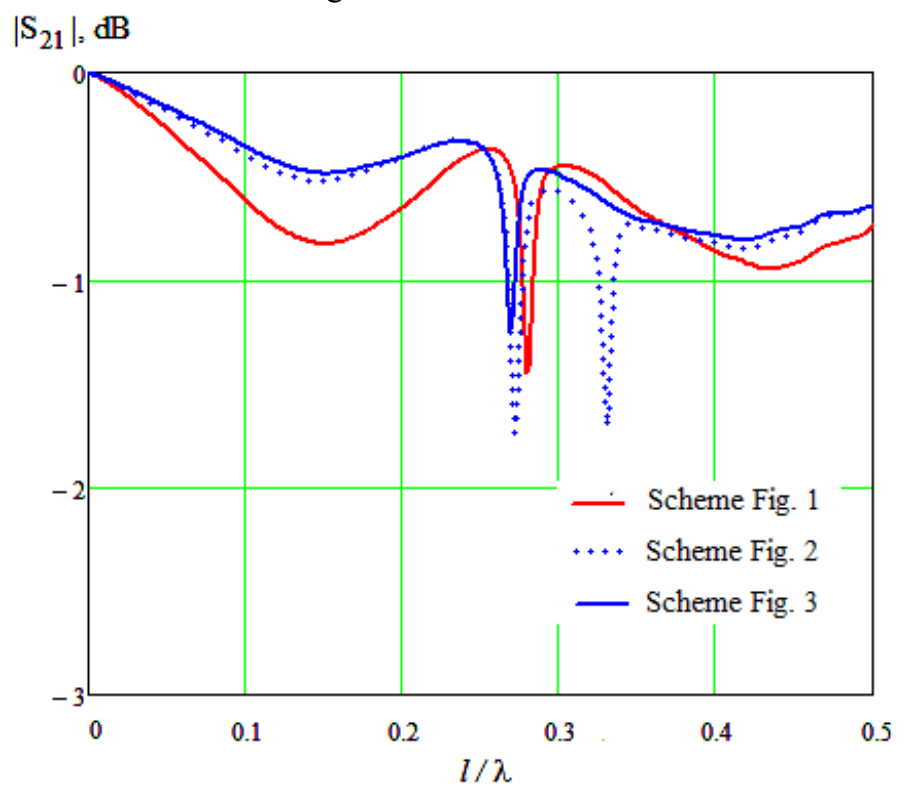

Fig. 10. The experimental dependence of insertion loss of a segment of a split strip line (diagram Fig. $1,2$ and 3$)$ at the ratio of the length to the wavelength of the affecting signal in free space.

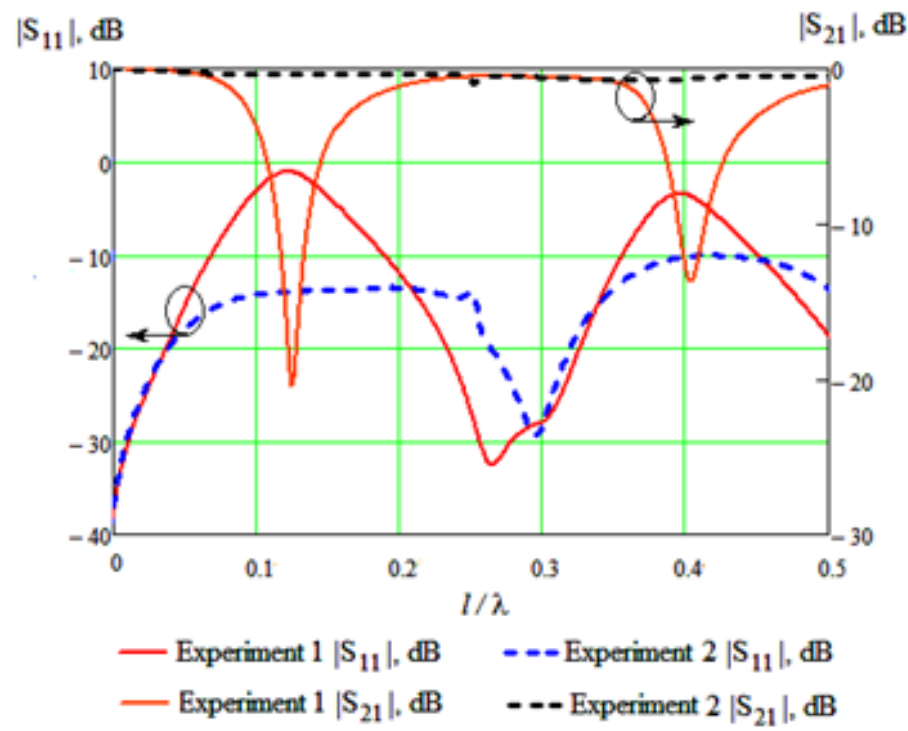

Fig. 11. Experimental dependences of insertion and return loss of SSL segment (circuit of Fig. 4 experiment 1, Fig. 5 - experiment 2 ) at the ratio of the length to the wavelength affecting the signal in free space. 
Fig. 12 shows the dependence of the insertion phase shift in circuits Fig. 4 and Fig. 5. It should be noted that the phase $\operatorname{shift} \arg \left(S_{21}\right) \rightarrow 90 \mathrm{deg}$ at $1 / \lambda \rightarrow 0$ in contrast to the equality of the phase shift is zero at $1 / \lambda \rightarrow 0$ in the single strip line with the T-wave.

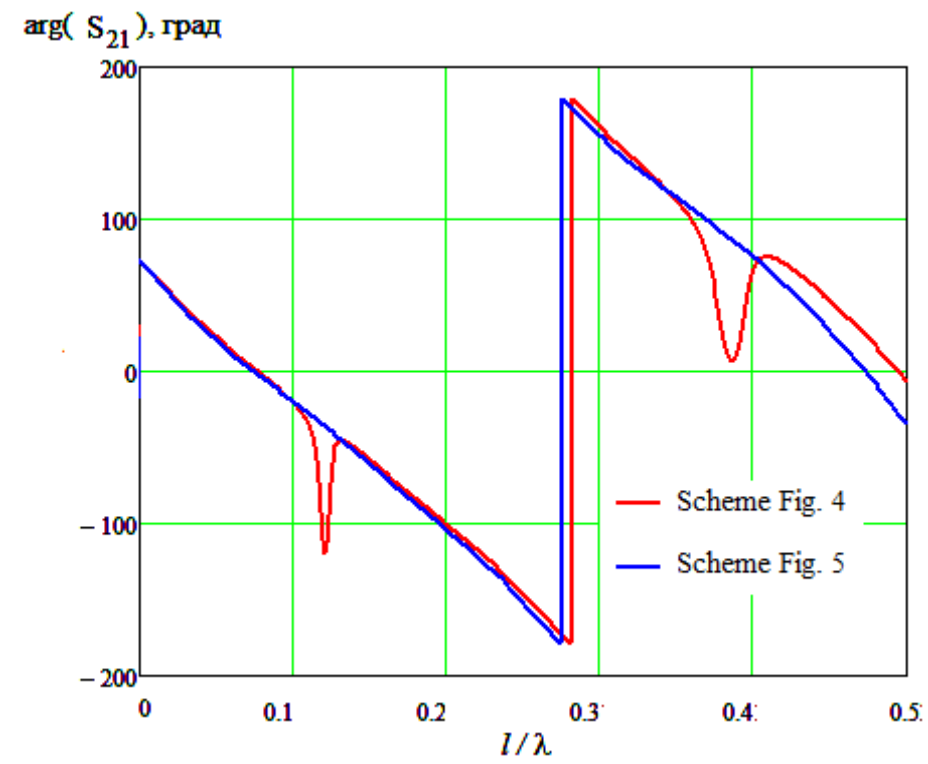

Fig. 12. The dependence of the insertion phase shift of a SSL segment.

\section{Discussion}

The obtained results allow to assert that the basic SSL sections in a prescribed restricted range of the length to the wavelength ratio affecting the signal in free space $1 / \lambda$ have fairly low insertion loss (about $0,5 \mathrm{~dB}$ ).

Note that in the links with the scheme of inclusion of the lines as in Fig. 1, 2 and 3 the strip 2 is present and it is completely DC isolated with strip 1, but it is involved in the transmission of power of microwave oscillations.

The presented and investigated links in Fig. 2, 3 and 5 are potentially adjustable, since the additional strip 3 can be shunted by the lumped capacity of the regulating elements.

At the same time, as follows from analysis of experimental dependencies of insertion losses of split strip line segment (circuits Fig. 1, 2 and 3) from ratio of segment length to wavelength of acting signal, characteristic impedance of strip structure is changed. The diagram of such segment is shown in Fig. 6.

The use of SSL segments in devices that require an equivalent transmission line with variable characteristic impedance, such as in impedance transformers, is advisable. This is especially true with large differences in the elements to be matched. The considered options of links on the basis of strip structure with an additional strip (fig. 9) as it is visible from comparison of frequency characteristics in Fig. 11, represent an equivalent of the coupled lines with changeable coefficient of coupling on electric field (on capacity) that can be the useful tool at design of the directional couplers and filters of various types.

\section{Conclusion}

The proposed type of split strip lines, allows you to design different devices based on the equivalent transmission line with variable characteristic impedance and coupling coefficient between the lines. 


\section{Acknowledgment}

The work was financially supported by the Ministry of science and higher education of the Russian Federation on the draft 14.577.21.0279 of 26.09.2013, ID RFMEFI57717X0279. The measurements were conducted at the equipment Center for collective use "Pulse" TUSUR.

\section{References}

1. B.A. Belyaev, A.A. Leksikov, V.F. Shabanov, and A.M. Serzhantov, Physical principles of the design of electrically controllable microstrip devices. Russian Physics Journal. 51. № 9. P. 919-929 (2008)

2. A.M.Serzhantov, K.V. Lemberg, Investigation of a liquid crystal tunable phase shifter / 2010 20th International Crimean Conference Microwave and Telecommunication Technology, Conference Proceedings 2010 20th International Crimean Conference Microwave and Telecommunication Technology. Sevastopol, Crimea. P. 702-703 (2010)

3. V.V. Pleskachev, I.B. Vendik, Tunable microwave filters based on ferroelectric capacitors / 13th International Crimean Conference Microwave and Telecommunication Technology, 2003. CriMiCo 2003. P. $468-470$. DOI: 10.1134/S1063785013090241 (2003)

4. Makoto Tsutsumi and Kensuke Okubo, Effect of stubs on ferrite microstrip line magnetized to wave propagation / 2009 Asia Pacific Microwave Conference. P. 1234 1237 (2009)

5. Moussavou, A.-G., Sauleau, R., Mahdjoubi, K., Deputier, S., Guilloux-Viry, M., and A. Perrin, Study of planar transmission lines printed on a ferroelectric thin film: Optimum tunability and figure of merit. 2006 First European Conference on Antennas and Propagation. DOI:10.1109/eucap. 4585018 (2006)

6. P.A. Vorob'ev., N.D. Malyutin, V.N. Federov Quasi-T-waves in devices using coupled strip lines with unbalanced electromagnetic coupling. Radio Engineering and Electronic Physics. V. 27. № 9. P. 38-44 (1982)

7. G.I. Zysman, A.K. Jonson. Coupled transmission line networks in an inhomogeneous dielectric medium. IEEE Trans, on MTT. 1969. Vol. MTT-17, № 10. - P. 753-759.

8. Vincent F. Fusco. Microwave circuits. Analysis and Computer Design. Prentice-Hall International (UK) Ltd (1987) 